\title{
Alterations of Bacteroides sp., Neisseria sp., Actinomyces sp., and Streptococcus sp. populations in the oropharyngeal microbiome are associated with liver cirrhosis and pneumonia
}

Haifeng $\mathrm{Lu}^{1+}$, Guirong Qian ${ }^{2+}$, Zhigang Ren ${ }^{1}$, Chunxia Zhang ${ }^{3}$, Hua Zhang ${ }^{1}$, Wei Xu' ${ }^{1}$, Ping Ye ${ }^{1}$, Yunmei Yang ${ }^{3}$ and Lanjuan $\mathrm{Li}^{1 *}$

\begin{abstract}
Background: The microbiomes of humans are associated with liver and lung inflammation. We identified and verified alterations of the oropharyngeal microbiome and assessed their association with cirrhosis and pneumonia.

Methods: Study components were as follows: (1) determination of the temporal stability of the oropharyngeal microbiome; (2) identification of oropharyngeal microbial variation in 90 subjects; (3) quantitative identification of disease-associated bacteria. DNAs enriched in bacterial sequences were produced from low-biomass oropharyngeal swabs using whole genome amplification and were analyzed using denaturing gradient gel electrophoresis analysis.

Results: Whole genome amplification combined with denaturing gradient gel electrophoresis analysis monitored successfully oropharyngeal microbial variations and showed that the composition of each subject's oropharyngeal microbiome remained relatively stable during the follow-up. The microbial composition of cirrhotic patients with pneumonia differed from those of others and clustered together in subgroup analysis. Further, species richness and the value of Shannon's diversity and evenness index increased significantly in patients with cirrhosis and pneumonia versus others ( $p<0.001$, versus healthy controls; $p<0.01$, versus cirrhotic patients without pneumonia). Moreover, we identified variants of Bacteroides, Eubacterium, Lachnospiraceae, Neisseria, Actinomyces, and Streptococcus through phylogenetic analysis. Quantitative polymerase chain reaction assays revealed that the populations of Bacteroides, Neisseria, and Actinomycetes increased, while that of Streptococcus decreased in cirrhotic patients with pneumonia versus others ( $p<0.001$, versus Healthy controls; $p<0.01$, versus cirrhotic patients without pneumonia).
\end{abstract}

Conclusions: Alterations of Bacteroides, Neisseria, Actinomyces, and Streptococcus populations in the oropharyngeal microbiome were associated with liver cirrhosis and pneumonia.

Keywords: Whole genome amplification (WGA), Oropharyngeal microbiome, Pneumonia, Cirrhosis, Denaturing gradient gel electrophoresis (DGGE)

\footnotetext{
* Correspondence: ljli@zju.edu.cn

${ }^{\dagger}$ Equal contributors

${ }^{1}$ State Key Laboratory for Diagnosis and Treatment of Infectious Diseases,

Collaborative Innovation Center for Diagnosis and Treatment of Infectious

Diseases, The First Affiliated Hospital, College of Medicine, Zhejiang

University, 79 Qingchun Road, Hangzhou 310003, People's Republic of China

Full list of author information is available at the end of the article
} 


\section{Background}

Liver disease and associated complications represent a major healthcare burden in China. Pneumonia is a far more common complication in patients with decompensated cirrhosis. Increasing awareness of the role of the microbiome of humans in the progression of liver [1] and lung inflammation $[2,3]$ raises the importance of assessing the composition of the respiratory microbiome as well as the nature of disease-induced changes caused by these microbiomes during disease progression. Current treatment strategies for pneumonia are based on the routine identification of cultured bacteria, including the isolation of clinically significant bacterial species from sputum. However, the upper and lower respiratory tracts harbor a vast range of commensal and potentially pathogenic bacteria that forms an indispensable part of the human microbiome [4]. These organisms live in a complex, yet balanced relationship, and therefore manipulation of one may affect the other members of the community. Although the presence of pathogens is a prerequisite for infections and alterations of the community, which may lead to overgrowth and invasion, it is a key factor leading to infection [5].

Studying the microbial variations of patients with pneumonia is challenging, because sampling the lung microbiome requires an invasive surgical procedure that may harm subjects. The oropharynx serves the respiratory and digestive systems and is colonized by bacterial pathogens that affect healthy or immunocompromised individuals [6]. The importance of the microbial composition of the oral and respiratory tracts is increasingly considered as a source of biomarkers to facilitate noninvasive detection of disease $[7,8]$. A better understanding of the significance of variations of the oropharyngeal mucosal microbiome in preclinical conditions and disease may provide insights into selective oropharyngeal decontamination that is used to prevent susceptible patients from contracting pneumonia [9]. Moreover, samples are acquired with minimal disturbance of the microbiome, and the technique exposes patients to minimal risk.

Denaturing gradient gel electrophoresis (DGGE) is a DNA fingerprinting technique used to accurately assess the members of a microbial community by generating patterns or profiles of genetic diversity [10]. DGGE facilitates rapid analyses and comparisons of microbial communities. Moreover, polymerase chain reaction (PCR) amplicons are isolated from the DGGE profile, which can be further amplified and sequenced $[10,11]$. Therefore, it is a preferred technique for studies of the composition, structure, and stability of complex microbial communities. However, the low biomass obtained from oropharyngeal swabs impedes the application of the DGGE technique for characterizing microbial populations of the oropharyngeal mucosa.
Whole genome amplification (WGA) using phi29 DNA polymerase overcomes insufficient sample sizes [12]. For example, numerous studies of microbial ecology show that WGA using phi29 DNA polymerase enriches environmental template DNAs with the highest amplification efficiency $[13,14]$, and the amplified DNA can be used to characterize the microbial community structure in lowbiomass environments [15-17]. Here, we applied phi29 DNA polymerase to generate sufficient quantities of DNA from oropharyngeal mucosal swabs to analyze and monitor the microbial community associated with liver cirrhosis and pneumonia, which was accompanied by further DGGE analysis of the V3 region of the $16 \mathrm{~S}$ rDNA. We aimed to conduct a preliminary assessment of the variations of the predominant oropharyngeal mucosal among cirrhotic patients with or without pneumonia and healthy controls as well as the microbial alterations during the follow-up. Our ultimate goal was extend our knowledge of the contributions of the respiratory microbiome in the human lung in health and disease.

\section{Methods}

\section{Study design, patients, and samples}

From September 2011 to August 2012, the study recruited 90 subjects, including 30 with hepatitis B virus (HBV)-decompensated cirrhosis with confirmed pneumonia (Group CI), 30 with HBV-decompensated cirrhosis without respiratory infection (Group CC), and 30 healthy adult controls matched for age and sex to the cirrhotic group (Group HC). The clinical characteristics collected from medical records of the subjects are shown in Table 1. A subset of subjects was randomly chosen to provide swab specimens every 5 days within the 3 -week follow-up period after a positive swab confirmation. The follow-up study included 12 patients with HBV-decompensated cirrhosis with pneumonia, 10 without respiratory infection, and 10 healthy controls. None of the controls had a detectable infection during the follow-up. The patients fulfilled the criteria as follows: (i) liver cirrhosis detected using ultrasound with or without pneumonia, which was confirmed using computerized tomography of the chest; (ii) physician's diagnosis of ascites, gastrointestinal bleeding, and malnutrition; (iii) not treated with antibiotics, lactulose, prokinetic drugs, or proton pump inhibitors 3 weeks before hospitalization; (iv) absence of other organ dysfunction, except the liver, lung, and previous related surgery. Exclusion criteria included other infections such as peritonitis and bacteremia confirmed using ascites fluid and routine bacteriological analysis of blood. A physician supervised the collection of the first swab specimens of the unstimulated oropharynx within $2 \mathrm{~h}$ after the patients' hospitalization, and antibiotics were not administered. Written informed consent and questionnaires (Additional file 1: Table S1) were obtained from all subjects who 
Table 1 Subject characteristics

\begin{tabular}{llll}
\hline Characteristics & Group HC $(n=30)$ & Group CC $(n=30)$ & Group Cl $(n=30)$ \\
\hline Age & $62.80 \pm 3.19$ & $63.47 \pm 2.29$ & $65.23 \pm 3.42$ \\
Sex: Male/female & $23 / 7$ & $21 / 9$ & $22 / 8$ \\
Hospital days & $-{ }^{-a}$ & 10.5 & 25.5 \\
Primary blood parameters at first follow-up: & & & $14.60 \pm 18.47$ \\
WBC $\left(10^{9} / \mathrm{L}\right)$ & $8.48 \pm 1.13$ & $21.97 \pm 7.54$ & $136.02 \pm 79.39$ \\
CRP $(\mathrm{mg} / \mathrm{L})$ & $<10$ & $29.97 \pm 7.54$ & $32.88 \pm 3.31$ \\
ALB $(\mathrm{g} / \mathrm{L})$ & $50.19 \pm 2.84$ & $140.53 \pm 53.04$ & $117.55 \pm 30.27$ \\
ALT $(\mathrm{U} / \mathrm{L})$ & $18.77 \pm 7.65$ & $173.07 \pm 62.10)$ & $109.29 \pm 38.47$ \\
AST $(\mathrm{U} / \mathrm{L})$ & $17.90 \pm 5.26)$ & $49.03 \pm 16.63$ & $51.37 \pm 23.44$ \\
TBIL $(\mathrm{mol} / \mathrm{L})$ & $9.63 \pm 1.65$ & & 5 \\
Infection with & & - & 5 \\
Candida & - & - & 3 \\
Streptococcus pneumoniae & - & - & 3 \\
Mycoplasma pneumoniae & - & - & 5 \\
Pseudomonas aeruginosa & - & - & \\
Staphylococcus & - & & 5 \\
\hline
\end{tabular}

Mean \pm SD

WBC white blood cell, CRP C-reactive protein, $A L B$ albumin, $A L T$ alanine aminotransferase, $A S T$ aspartate aminotransferase, $T B I L$ total bilirubin ${ }^{\mathrm{a}}$ Not applicable

voluntarily underwent sample collection using oropharyngeal mucosal swabs. The Ethics Committee of the First Affiliated Hospital, School of Medicine, Zhejiang University approved this study.

\section{Sampling and DNA extraction}

Microbial samples were obtained from the posterior wall of the oropharynx using sterile Copan swabs (Copan Diagnostics Inc., California, USA). The swab sample was immersed in phosphate-buffered saline, transferred to the laboratory, shaken, centrifuged immediately, and the supernatant was discarded. The pellets were stored at $-80^{\circ} \mathrm{C}$ within an hour. Microbial DNA was extracted using a Qiagen Mini Kit (Qiagen, Hilden, Germany) following the manufacturer's instructions. The DNA was quantified using a Qubit 2.0 Fluorometer (Invitrogen, Carlsbad, CA, USA), and all oropharyngeal microbial DNAs were diluted to $3 \mathrm{ng} / \mu \mathrm{L}$ for WGA.

\section{Whole genome amplification (WGA)}

Bacterial genomic DNA was subjected to multiple displacement amplification using the GenomiPhi V2 DNA Amplification Kit [12] (GE Healthcare, Amersham Place, Buckinghamshire, UK) according to the manufacturer's protocol. After WGA, the amplicons were quantified using a Qubit 2.0 Fluorometer. Each sample was amplified in triplicate, and the three reaction products per sample were diluted $1: 10$ and stored at $-30{ }^{\circ} \mathrm{C}$.

\section{PCR amplification of the 16S rDNA V3 region}

The V3 variable region of $16 \mathrm{~S}$ rDNA was amplified using a hot-start touchdown protocol with primers specific for the conserved regions of the 16S rRNA gene [18]. The reaction mixture contained $400 \mathrm{ng}$ of genomic DNA, 25 pmol of each primer, $0.2 \mu \mathrm{M}$ dNTPs, $1 \times$ Ex Taq buffer, and $2.5 \mathrm{U}$ of Ex Taq polymerase (Takara, Dalian, China), and the final volume was adjusted to $50 \mu \mathrm{L}$ with sterile deionized water. To minimize hetero duplex formation, five-cycle reconditioning PCR was conducted using $5 \mu \mathrm{L}$ of amplification mixture in a fresh reaction mixture as previously described [19]. The reamplified products $(25 \mu \mathrm{L})$ were analyzed using DGGE.

\section{DGGE profiling}

Parallel DGGE profiling was performed as previously described $[11,20]$. Electrophoresis was conducted at a constant $70 \mathrm{~V}$ at $60{ }^{\circ} \mathrm{C}$ for approximately $16 \mathrm{~h}$. DGGE profiles were processed using BioNumerics software version 6.01 (Applied Maths, St-Martens-Latem, Belgium) in a multistep procedure following the manufacturer's instructions. Parameters for allocating band-classes were set according to Joossens et al. [20]. Cluster analysis of DGGE profiles used the unweighted pair-group method with an arithmetic mean (UPGMA) based on the Dice similarity coefficient [21]. Multidimensional scaling (MDS) and principal component analysis (PCA) were conducted according to their respective manuals in BioNumerics software. Past software was used to derive the 
Shannon H scores (diversity index), Shannon's evenness index, and species richness [22].

The samples were fractionated using 19 gels, and three standard reference lanes were included on the sides and in the middle of each DGGE gel to allow comparisons among gels using BioNumerics software. We excised as many bands as possible from each gel. Gel slices were placed in sterile Eppendorf tubes, washed three times in TE buffer (10 mM Tris- $\mathrm{HCl}, 1 \mathrm{mM}$ EDTA, $\mathrm{pH}$ 8.0), disrupted, and incubated in $50 \mu \mathrm{L}$ of TE buffer for $30 \mathrm{~min}$ at $80{ }^{\circ} \mathrm{C}$. DNA in $5 \mu \mathrm{L}$ of buffer solution was used as a template for PCR re-amplification with universal bacterial primers F357 + GC clamp and R518 as described above for DGGE. Amplicons were analyzed using DGGE, excised until a single band was obtained, stored at $-20^{\circ} \mathrm{C}$, and verified according to their reference site in DGGE gels by comparing gels using BioNumerics software. Two or three amplicons from the same band-class were selected and purified using a QIA quick PCR purification Kit (Qiagen, Hilden, Germany), ligated to pGEM-T Easy Vector DNA (Promega, Madison, WI, USA), and used to transform competent Escherichia coli DH5. Positive colonies were verified and sequenced (Invitrogen, Shanghai, China). The sequences were deposited into the European Nucleotide Archive (ENA) database (Submission ID: Hx200004778).

Sequence similarities were determined using BLAST to search the GenBank DNA database [14]. Based on the BLAST results, reference sequences of phylogenetic neighboring species (97\% similarity) were included for cluster analysis according to multiple sequence alignments generated using MEGA5 software (Molecular Evolutionary Genetics Analysis version 5) [10].

\section{Quantitative PCR}

Quantification of the bacterial species of interest in each original (non-WGA) sample DNA was performed using qPCR as described previously [11, 20]. The primers (Shanghai Invitrogen Biotechnology Limited Company, Shanghai, China) and annealing temperatures are shown in Additional file 2: Table S2. Quantification of Lachnospiraceae and Bacteroides was performed using a Taqman assay, and other species-specific assays employed SYBR Green.

The copy number of bacterial species was determined by comparison with serially diluted plasmid DNA standards run on the same plate. The plasmid DNA standards were prepared from known concentrations of plasmid DNA [23]. The protocol to determine the detection limit was performed as described previously [24]. The abundances of bacterial species were expressed as $\log 10$ values per $10 \mathrm{ng}$ original DNA template.

\section{Statistical analysis}

Statistical analysis was conducted according to Joossens et al. [10]. Mann-Whitney tests were performed to compare differences in oropharyngeal microbiomes across the groups using SPSS version 17.0 for Windows (SPSS Inc., Chicago, IL, USA).

\section{Results}

The applicability, feasibility, and validity of the WGA method to enrich microbial DNA

We first analyzed the applicability of the WGA method for characterizing oropharyngeal microbial DNA samples. We compared the DGGE profiles between WGAamplified DNA (Fig. 1a, lanes A1, B1, C1, and D1) and the original DNA (Fig. 1a, lanes A0, B0, C0, and D0) and found that the WGA-positive samples comprised more bands than those of WGA-negative samples, suggesting that the WGA method was capable of enriching oropharyngeal microbial DNA samples for DGGE analysis.

We then determined the reproducibility of the WGA method. Two fecal microbial DNAs were diluted 1:100 (3 $\mathrm{ng} / \mu \mathrm{L}$ ) and amplified in triplicate using the WGA method. The DGGE profile analysis indicated that the WGA method amplified fecal DNA (Fig. 1b, lanes A1-3 and $\mathrm{B} 1-3)$, the most predominant bands were similar to those of the original fecal DNA (Fig. 1b, lanes A0 and B0), and the results were reproducible. These results prove that the WGA method effectively enriched low concentrations of microbial DNA suitable for DGGE analysis.

The validity of the WGA method was further characterized using DGGE profiling and cluster analysis. Seven oropharyngeal microbial DNA samples were amplified in duplicate using WGA. Cluster analysis showed that the DGGE profiles were $>90 \%$ similar between duplicates (Fig. 1c), suggesting the validity and reproducibility of the WGA method for preparing small quantities of DNA for DGGE analysis.

\section{Determination of the temporal stability of the oropharyngeal microbiome in a 3- week follow-up study} To investigate the associations between the oropharyngeal microbiome and disease, we first confirmed the temporal stability of oropharyngeal microbiomes of the $\mathrm{HC}$ and $\mathrm{CC}$ groups during a follow-up period. We chose randomly 32 subjects, including 10 volunteers from the $\mathrm{HC}$ group (three times), 10 patients from the CC group (three times, most were discharged from our hospital before the fourth appointment.), and 12 patients from the CI group (four times). The first sample from the patients in the CI group were before antibiotic treatments, the second samples were during antibiotic treatments, and the third and fourth were after antibiotic treatments.

The DGGE profiles and cluster analysis demonstrated that three follow-up samples of each individual from the $\mathrm{HC}$ or CC groups $(n=10)$ clustered together, respectively (Fig. 2a). These results suggest that the oropharyngeal mucosal microbiome of each control individual 

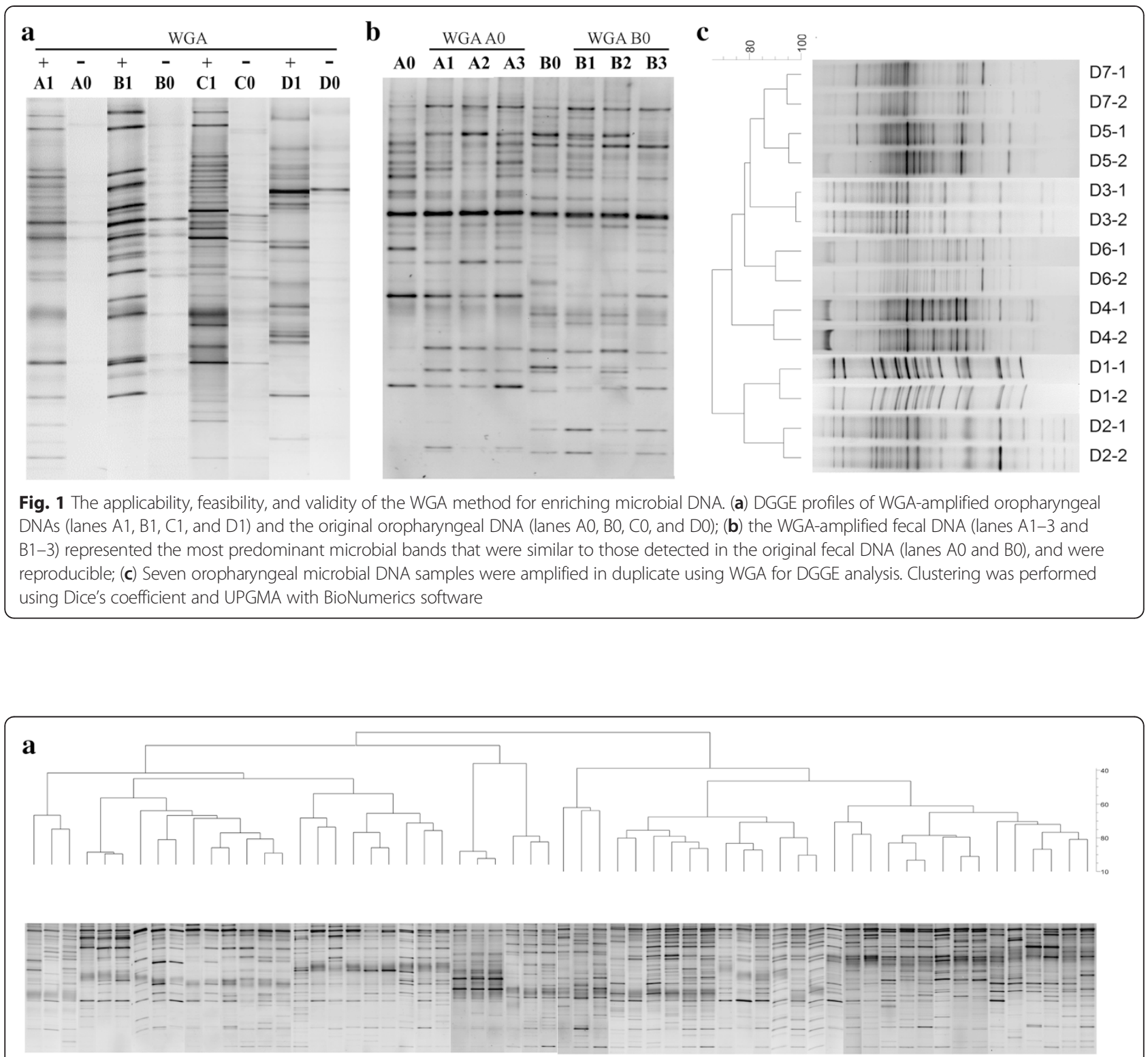

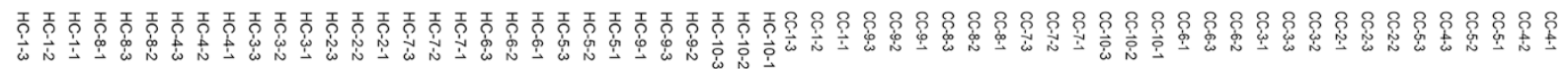

b

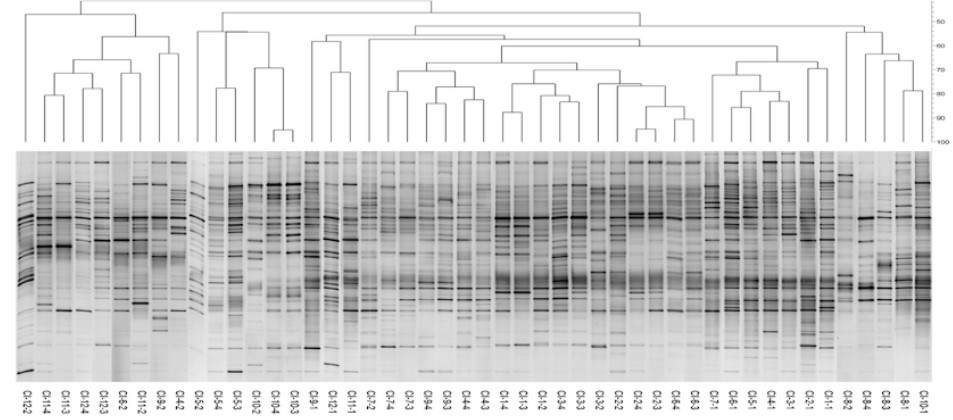

c

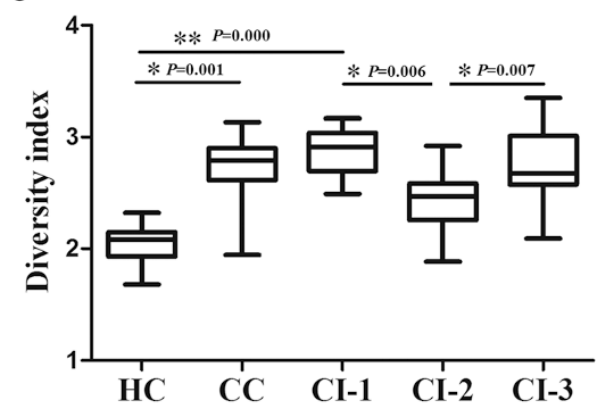

Fig. 2 Confirmation of the temporal stability of the oropharyngeal microbiome in a 5-day follow-up study. Cluster analysis of DGGE profiles of oropharyngeal bacteria in groups $\mathrm{HC}(\mathbf{a}), \mathrm{CC}(\mathrm{a})$, and $\mathrm{Cl}(\mathrm{b})$. Cluster analysis was performed using Dice's coefficient and UPGMA. Lanes were designated by patient number $(1,2,3,4)$, and visit number (1, 2, 3, and 4).Cln-1, before antibiotic treatment; Cln-2, during antibiotic treatment; Cln-3 and -4, after antibiotic treatment. The diversity index of these follow-up samples is shown (c). ${ }^{*} p<0.01 ;{ }^{* *} p<0.001$ 
exhibited relatively stable patterns during the follow-up (Fig. 2a). In contrast, because of antibiotic treatment, four follow-up samples from patients with pneumonia from the CI group $(n=12)$ did not cluster together (Fig. 2b). However, the DGGE profiles of each patients with pneumonia were similar between their third and fourth visits (Fig. 2b), suggesting that the oropharyngeal microbiome was relatively stable in patients after antibiotics treatments.

Moreover, the diversity of the oropharyngeal microbiome among different groups was calculated using Past software. The predominant microbiome of patients with pneumonia from group $\mathrm{CI}$ had the highest diversity (CI-1), followed by control patients with liver cirrhosis (group CC) (Fig. 2c). The diversities of groups $\mathrm{CI}$ and $\mathrm{CC}$ were higher compared with that of the group HC. Notably, oropharyngeal microbial diversity was decreased in group CI-2 during antibiotic treatments versus group CI-1 before antibiotic treatments.

To investigate oropharyngeal microbial variation and identify the key bacteria associated with liver cirrhosis and pneumonia, we analyzed the DGGE profiles of the 90 subjects.

\section{Cluster analysis of DGGE profiles}

Cluster analysis of DGGE profiles indicates that almost all individuals in each group (except for three samples from the group $\mathrm{CC}$ ) clustered together, suggesting that the microbial composition of each individual in the same group was similar to the others and that the microbial composition of patients in the group CI differed from those of both control groups. Notably, the DGGE profiles of all patients in group CI clustered together at high UPGMA coefficient values ranging from 57.7 to $94.0 \%$ (average, $82.30 \pm 9.85$, Fig. 3a). These results were confirmed using MDS analysis (Fig. 3b) and PCA (Fig. 3c). For example, note the overlap of symbols representing the microbiomes of patients infected with the same pathogen in the group CI.

\section{Analysis of microbial diversity}

We used Past software to assess the microbial diversity of the oropharyngeal mucosa using Shannon's diversity index, Species richness, and Shannon's evenness index. The values of Shannon's diversity index, Species richness, and Shannon's evenness index were obviously higher in group CI compared with those in groups $\mathrm{CC}$ and $\mathrm{HC}(p<0.01)$ (Fig. 3d). Further,

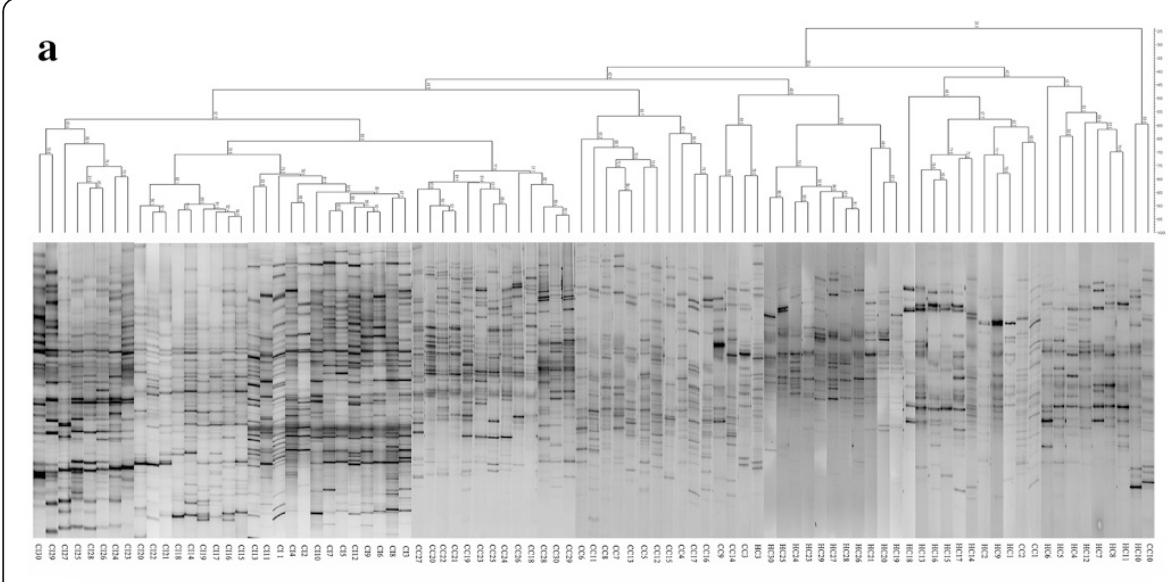

b
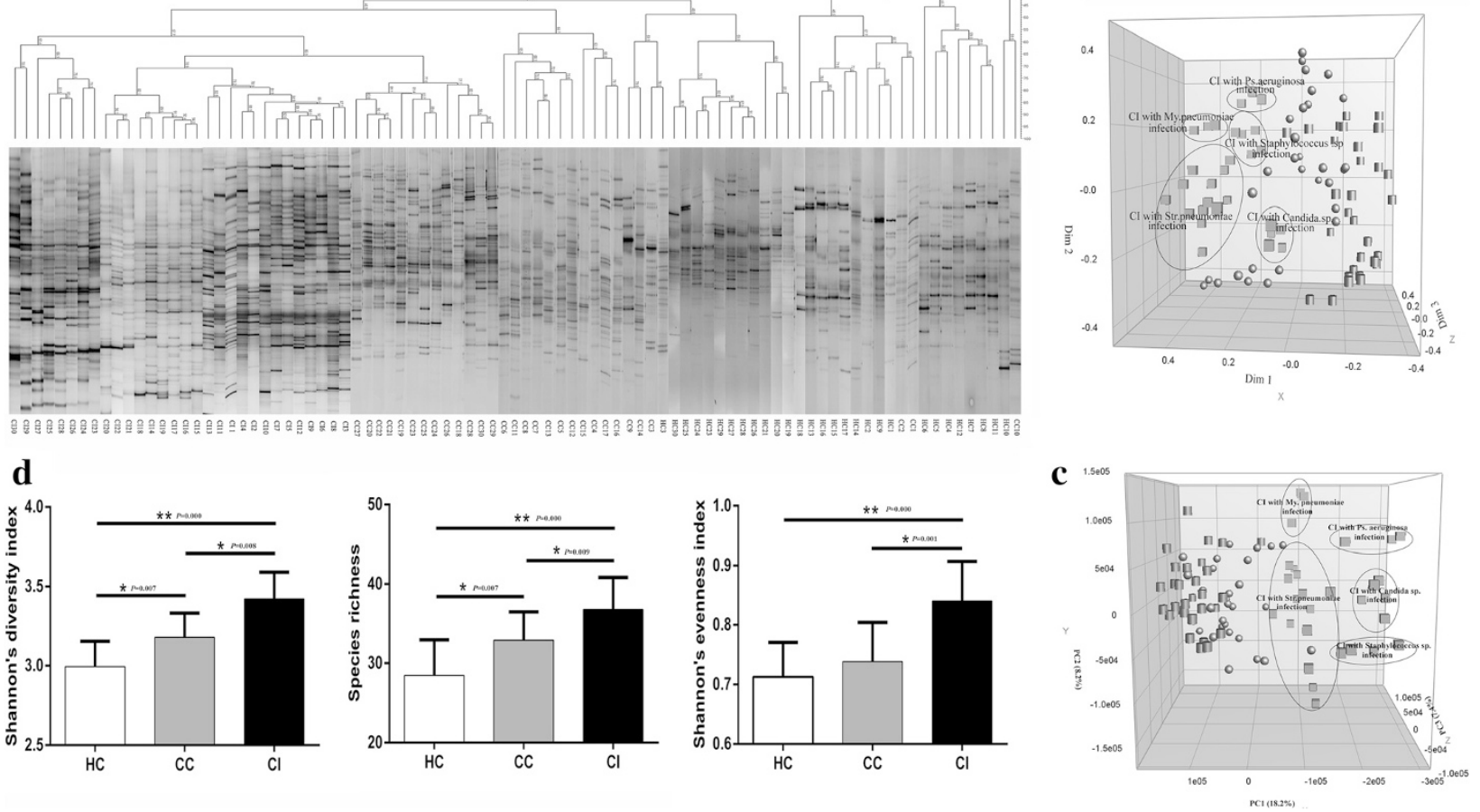

Fig. 3 Cluster and diversity analyses of DGGE profiles of the oropharyngeal mucosal microbiomes of 90 PCR-DGGE profiles. (a) Cluster analysis of DGGE profiles of the oropharyngeal mucosal microbiome; (b) Multidimensional scaling analysis (MDS) based on the predominant oropharyngeal bacterial PCR-DGGE profiles. The plot is an optimized three-dimensional representation of the similarity matrix obtained using BioNumerics software, and the $x-, y$-, and z-axes represent three different dimensions (Dim 1, Dim 2, and Dim 3, respectively). (c) Principal component analysis (PCA) based on bacterial PCR-DGGE profiles. The plots were reoriented to maximize the variation among lanes along the first three principal components (contributions: 18.2, 8.2, and 7.4\%, respectively) obtained using BioNumerics software. Cubes, group Cl; spheres, group CC; cylinders, group HC. (d) Comparison of the diversity index, species richness, and evenness index of oropharyngeal mucosal microbiomes. ${ }^{*} p<0.01$; ${ }^{* *} p<0.001$ 
Shannon's diversity index and Species richness were higher in group CC compared with group HC $(p<0.01)$.

\section{Phylogenetic analysis of DNAs isolated from DGGE profiles} In the 90 PCR-DGGE profiles analyzed in this study, 39 band-classes were identified (Fig. 4a). Firmicutes (20 bandclasses) was the most common phylum, followed by Actinobacteria (8 band-classes), Bacteroidetes (4 band-classes), Proteobacteria (4 band-classes), and Fusobacteria (3 bandclasses) (Fig. 4b). Seven band-classes were highly prevalent (median intensity in at least one of the groups was $>2 \%$ ), including band-classes 30.1, 33.0, and 65.6 of Streptococcus, band-class 8.3 of Fusobacterium, band-class 59.4 of Veillonella, as well as band-classes 80.6 and 63.0 of Actinomyces, in which there was little variance among band-classes 65.6, 59.4, and 80.6 (Additional file 3: Table S3).

\section{Identification of bacterial species that account for the variation in the oropharyngeal microbiome}

To identify the key bacterial species that shifted composition, we calculated the intensity and the frequency of each band in the 90 PCR-DGGE profiles and analyzed the variation of each band-class. We found that the intensities of 19 band-classes differed significantly (Fig. 5a), and the frequencies of 14 band-classes varied (Fig. 5b) among the three groups. According to our analysis of the intensities and frequencies of the variable band-classes, six key band-classes were identified that reflected the differences between the CI and each control group. The abundances of five band-classes 4.8 (Bacteroides sp.), 36.8 (Eubacterium sp.), 43.3 (Lachnospiraceae sp.), 54.9 (Neisseria sp.), and 63.0 (Actinomyces sp.) were much higher in the group CI compared closely with those in each control group, while the abundance of band-class 30.1 (Streptococcus sp.) in group CI was significantly lower compared with each control group $(P<0.017$ with modified Bonferroni correction the $P$-values were shown in Supplementary Table S3.)

To assess the effects of liver cirrhosis on band-class distribution, we analyzed the differences in the abundances of the band-classes between groups $\mathrm{CC}$ and $\mathrm{HC}$ and found that five band-classes, including 26.5 (Bulleidia sp.), 33.0 (Streptococcus sp.), 34.5 (Haemophilus sp.), 45.4 (Lactobacillus sp.), and 90.9 (Olsenella sp.) were higher in abundance in group $\mathrm{CC}$ versus group $\mathrm{HC}$, while the abundances

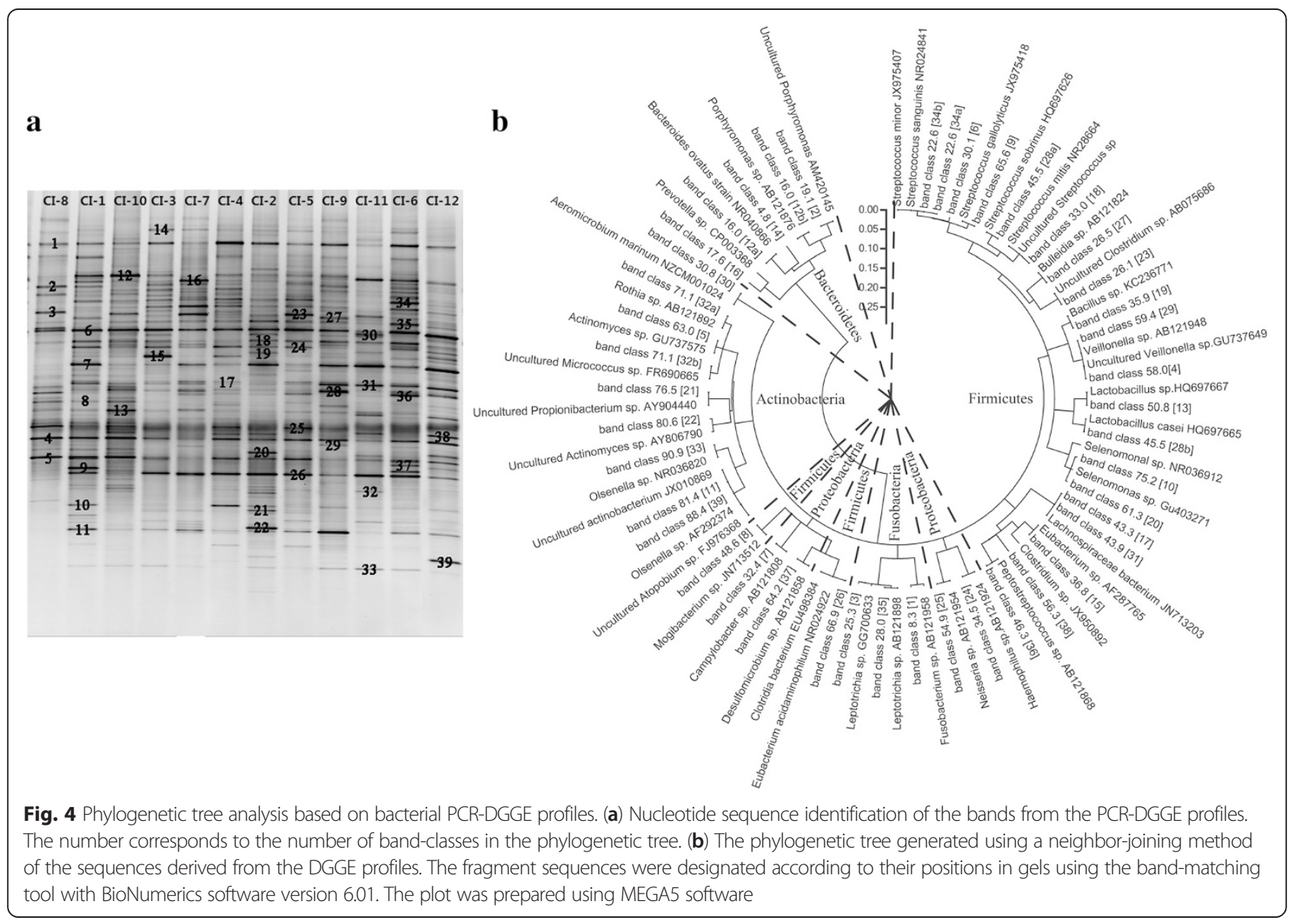




\section{a}

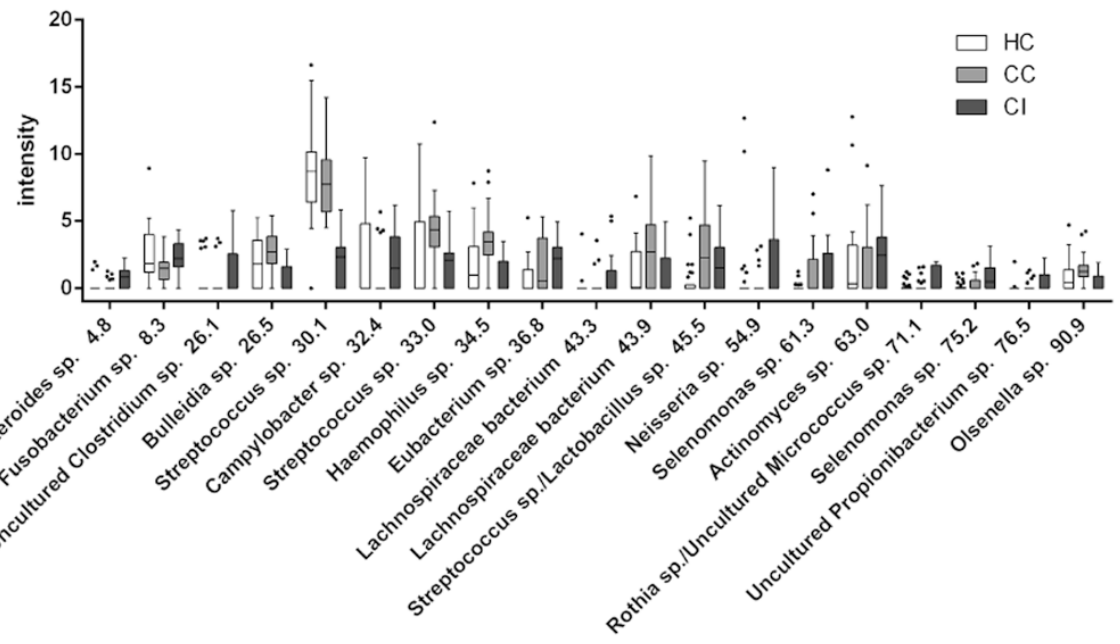

b

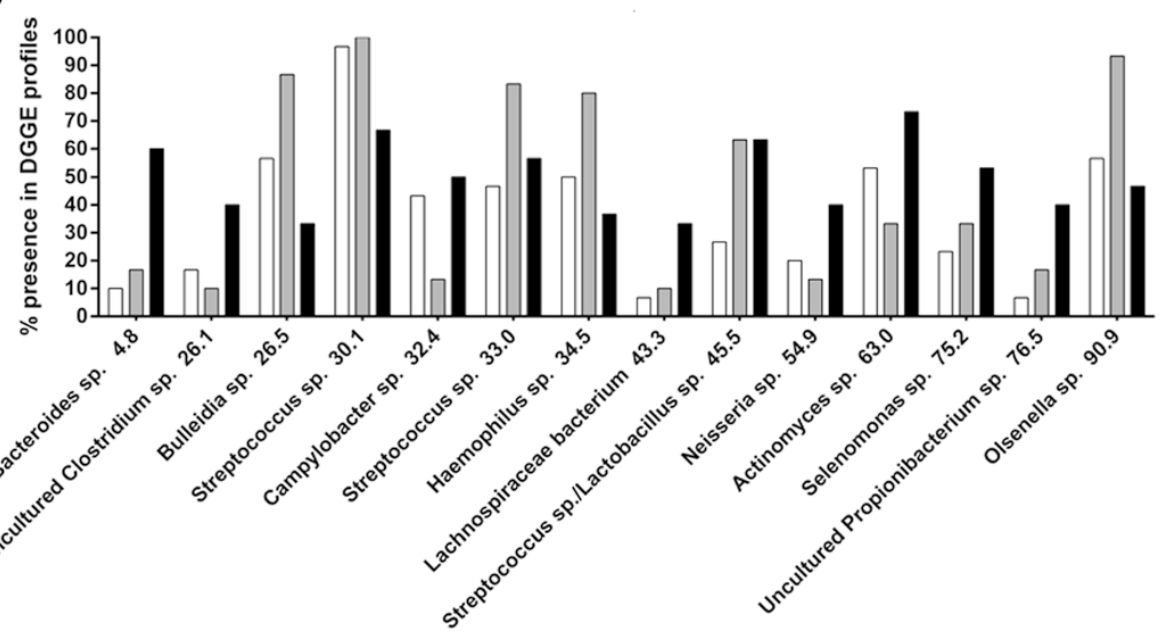

Fig. 5 Identification and analysis of the key band-classes based on bacterial PCR-DGGE profiles of the groups. (a) Comparisons intensities of the key band-classes based on bacterial PCR-DGGE profiles of the different groups. (b) Analysis of the frequencies of key band-classes based on bacterial PCR-DGGE profiles of the different groups. ( $P$-values for the comparison between the groups were shown in supplementary table S3)

of band-classes 32.4 (Campylobacter sp.) and 63.0 (Actinomyces sp.) decreased in group CC versus group $\mathrm{HC}$.

\section{Quantitative analysis of the disease-associated differences in the original (non-WGA) DNA}

To verify the key bacteria present in infected subjects, which were associated with oropharyngeal microbial variation, quantitative PCR was used to analyze the original (non-WGA) DNA samples using bacterial speciesspecific primers. The abundances of Bacteroides sp., Neisseria sp., and Actinomycetes sp. and those of Streptococcus sp. in the oropharyngeal mucosa of group CI were higher and lower, respectively, compared with those of groups CC and HC (3.23, IQR 2.07-4.10 versus 1.08, IQR 0-1.91 and 1.97, IQR 1.27-2.85 for Bacteroides sp.; 1.78, IQR 1.33-3.60 versus 0 , IQR $0-1.10$ and
0 IQR 0-0 for Neisseria sp; 2.42, IQR 0-3.77 versus 0, IQR 0-3.02 and 0, IQR 0-3.18 for Actinomycetes sp.; 3.26, IQR 2.48-4.66 versus 4.74, IQR 4.08-6.03 and 4.03, IQR 3.01-5.44 for Streptococcus sp.) $(p<0.05)$ (Fig. 6). The abundances of Bacteroides sp. and Streptococcus mitis were higher in group $\mathrm{CC}$ versus group $\mathrm{HC}$ $(p<0.05)$.

\section{Discussion}

WGA enables discovery of genomic information in the field of microbial ecology that was not previously accessible [25]. In the present study, each swab of microbial DNA was amplified in triplicate using phi29 V2 DNA polymerase and analyzed using PCR-DGGE. The sequences of 39 band-classes representing commensal bacterial species were found to differ and were identified using BLAST 


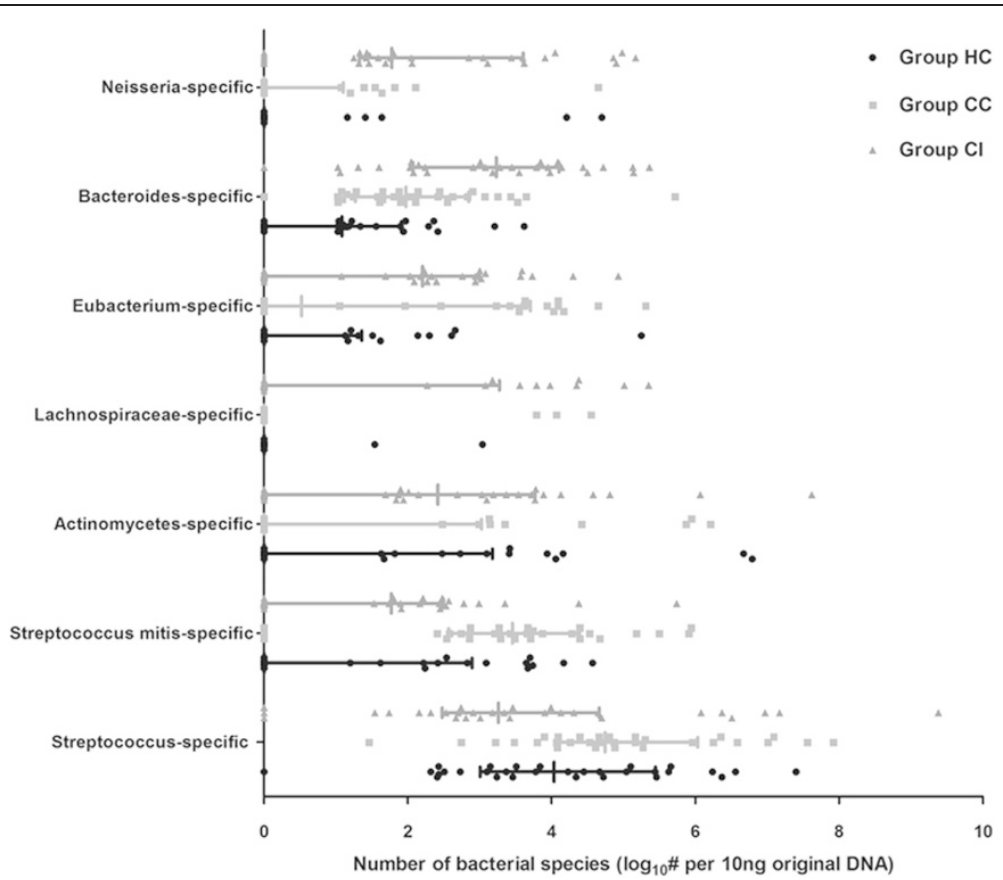

Fig. 6 RT-qPCR analysis of the key bacterial species accounting for variations in microbiome compositions. Number of key bacteria: log \# per $10 \mathrm{ng}$ original swab DNA. The figure shows the numbers of key bacteria, including Streptococcus, Streptococcus mitis, Actinomycetes, Lachnospiraceae, Eubacterium, Bacteroides, and Neisseria

to represent Streptococcus, Actinomyces, Neisseria, Prevotella, Rothia, Veillonella, Eubacteriaceae, and Fusobacteria. The oropharyngeal mucosal microbiome comprised Firmicutes, Actinobacteria, Bacteroidetes, Proteobacteria, and Fusobacteria, which is consistent with the results of others using high-throughput sequencing of the oral microbiome [26, 27].

The species listed above are always present at high frequency, typically in a balanced population in healthy individuals, and changes in their composition cause subsequent respiratory tract infections [28]. Therefore, the validity and reproducibility of the WGA method suggests that it is an appropriate technique for conducting subsequent PCRDGGE analysis to determine and monitor the predominant differences in oropharyngeal microbiota in a large cohort of patients with respiratory tract infections.

The human microbiome, including oropharyngeal bacteria, is an essential component of immunity that influences pathogenesis [29] and determines the body's physiological responses and susceptibility to disease [30]. Farrell and Zhang et al. [7] found that salivary microbiota serve as an informative source for discovering noninvasive biomarkers of pancreatic cancer and chronic pancreatitis. Here, our DGGE profiling results reveal considerable differences in the composition of oropharyngeal mucosal bacterial groups CI, CC, and HC. Further, increased diversity was detected in groups $\mathrm{CI}$ and $\mathrm{CC}$ compared with group $\mathrm{HC}$, particularly for group CI. It is important to note that quantitative PCR analysis verified that the abundances of Bacteroides sp., Neisseria sp., and Actinomycetes sp. were high compared with that of Streptococcus sp. in group CI versus groups CC and HC. Therefore, we conclude that alterations of the populations of these four bacterial species in the oropharyngeal microbiome were associated with liver cirrhosis and pneumonia.

The mechanisms responsible for alterations of the microbiome are multifactorial and complex. First, viral infection modifies the systemic effects of the host by modulating the host's immune response, which impairs mucosal immunity. Moreover, impaired local immunity and physical damage to the epithelium might enhance bacterial adherence and invasion. Accumulated evidence shows that infection is associated with polymicrobial interactions on mucosal surfaces that include commensal bacteria and exogenous pathogens [31]. For example, some pathogenic Candida species aggregate with Fusobacterium sp. [32], Actinomyces sp. [33], which may increase the colonization of mucosal epithelial cells.

Here, our quantitative PCR analysis indicates that the populations of Bacteroides sp., Neisseria sp., and Actinomycetes sp. increased dramatically and became the most prevalent species in group CI. These species originate in the oropharyngeal flora, and pathogenic and nonpathogenic Bacteroides sp. [34], Neisseria sp. [35] and Actinomycetes sp. [36] secrete outer-membrane vesicles that interact with neighboring cells through 
fusion or adherence. These microvesicles may impart a growth advantage, causing an imbalance in the composition of the oropharyngeal microbiota [37, 38] and subsequently impairing the barrier function of the mucosal biofilm, leading to the emergence of potentially pathogenic bacteria.

Moreover, host innate immune responses involve in competitive interactions between species and influence the structure and function of the flora [39]. Immune dysfunction is the most common clinical characteristic of patients with cirrhosis [40, 41] and is accompanied by alterations in the intestinal microbiota [42]. Intestinal microbes might interact with the microbiomes of other distant sites, including the respiratory tract and oral cavity [43] by activating the innate immune response. Therefore, we hypothesize that immune dysfunction might promote the growth of disease-associated oropharyngeal mucosal bacteria and subsequently cause an imbalance of oropharyngeal microbiota and enhanced susceptibility to infection by pathogens and facultative bacteria.

Moreover, the infection might inversely enhance the destruction of oropharyngeal microbiota. The destruction of oropharyngeal microbiota and infection might exert a synergetic effect on disease progression [31]. Strikingly, compared with group HC, the abundance of bacteria closely related to Streptococcus increased remarkably in group CC but decreased in group CI. In contrast, the population of bacteria closely related to Actinomyces decreased dramatically in group CC but increased in group CI. Bacterial colonization is determined by the ability to compete with coinhabitants of a niche [44]. Understanding the importance of interspecies interactions and intra-species genetic and phenotypic variation might serve to control disease progression and influence treatment [45].

\section{Conclusions}

The combination of WGA and DGGE analysis successfully monitored oropharyngeal microbial variations, and established that oropharyngeal microbiome of each subject maintained a relatively stable composition during the follow-up. However, it will be challenging to demonstrate a direct link between the species that populate the microbiota and the pathogenesis of pneumonia. Further studies using metagenomic approaches are required to identify the variations in more depth.

\section{Additional files}

Additional file 1: Table S1. Questionnaire: Follow-up data for subjects who participated in the investigation of the oropharyngeal microbiome.

Additional file 2: Table S2. The primers for $\mathrm{QPCR}$ in the study.

Additional file 3: Table S3. Median intensity of band classless that differ significantly among $\mathrm{HC}$ CC and $\mathrm{Cl}$ groups.

\section{Abbreviations}

WGA: Whole genome amplification; DGGE: Denaturing gradient gel electrophoresis; qPCR: Quantitative polymerase chain reaction; Group Cl: HBV decompensated cirrhotic patients with confirmed pneumonia; Group CC: HBV decompensated cirrhosis without any respiratory infection; Group HC: Healthy adult controls; UPGMA: The unweighted pair-group method with an arithmetic means; MDS: Multidimensional scaling; PCA: principal component analysis.

\section{Competing interests}

The authors declare that they have no competing interests.

\section{Authors' contributions}

$\mathrm{HL}$ and GQ helped design the study, collected and analyzed data, drafted the manuscript and writing the manuscript. ZR, $\mathrm{CZ}$ and $\mathrm{ZH}$ performed the experiments and acquired data. WX prepared the figures. PY and $Y Y$ had roles in subject recruitment and field investigation, and were involved in data analysis, interpretation. LL designed the study and revised the manuscript. All authors read and approved the final manuscript.

\section{Acknowledgments}

We acknowledge the physicians at the Department of Infectious Diseases in the First Affiliated Hospital, College of Medicine, Zhejiang University for their collaboration and collection of specimens. This study was supported by the National Basic Research Program (973) of China [Grant Nos. 2013CB531401, 2013CB531403], National Science and Technology Major Project [Grant No. Y2090363], Zhejiang Provincial Medical Scientific Research Foundation of

China [Grant No. 2012RCB020], and the Zhejiang Chinese Medicine

Administrative Bureau Project [Grant No. 2009ZA012].

\section{Author details}

'State Key Laboratory for Diagnosis and Treatment of Infectious Diseases, Collaborative Innovation Center for Diagnosis and Treatment of Infectious Diseases, The First Affiliated Hospital, College of Medicine, Zhejiang University, 79 Qingchun Road, Hangzhou 310003, People's Republic of China. ${ }^{2}$ Tonglu First People's Hospital, 338 Xuesheng Road, Tonglu, Hangzhou 311500, People's Republic of China. ${ }^{3}$ Department of Geriatrics, The First Affiliated Hospital, School of Medicine, Zhejiang University, 79 Qingchun Road, Hangzhou 310003, People's Republic of China.

Received: 8 January 2015 Accepted: 3 June 2015

Published online: 23 June 2015

\section{References}

1. Zeuzem S. Gut-liver axis. Int J Colorectal Dis. 2000;15(2):59-82.

2. Beck JM, Young VB, Huffnagle GB. The microbiome of the lung. Transl Res. 2012;160(4):258-66.

3. Forsythe P. Probiotics and lung diseases. Chest. 2011;139(4):901-8.

4. Charlson ES, Bittinger K, Haas AR, Fitzgerald AS, Frank I, Yadav A, et al. Topographical continuity of bacterial populations in the healthy human respiratory tract. Am J Respir Crit Care Med. 2011;184(8):957-63.

5. Bosch AA, Biesbroek G, Trzcinski K, Sanders EA, Bogaert D. Viral and bacterial interactions in the upper respiratory tract. PLoS Pathog. 2013;9(1):e1003057.

6. Segata N, Haake SK, Mannon P, Lemon KP, Waldron L, et al. Composition of the adult digestive tract bacterial microbiome based on seven mouth surfaces, tonsils, throat and stool samples. Genome Biol. 2012;13(6):R42.

7. Farrell JJ, Zhang L, Zhou H, Chia D, Elashoff D, et al. Variations of oral microbiota are associated with pancreatic diseases including pancreatic cancer. Gut. 2012;61(4):582-8.

8. Koren O, Spor A, Felin J, Fak F, Stombaugh J, et al. Human oral, gut, and plaque microbiota in patients with atherosclerosis. Proc Natl Acad Sci U S A. 2011;108 Suppl 1:4592-8.

9. Bergmans DC, Bonten MJ, Gaillard CA, et al. Prevention of ventilator-associated pneumonia by oral decontamination: a prospective, randomized, double-blind, placebo-controlled study. Am J Respir Crit Care Med. 2001;164:382-8.

10. Joossens M, Huys G, Cnockaert M, De Preter V, Verbeke K, et al. Dysbiosis of the faecal microbiota in patients with Crohn's disease and their unaffected relatives. Gut. 2011;60(5):631-7.

11. Lu H, He J, Wu Z, Xu W, Zhang H, et al. Assessment of microbiome variation during the perioperative period in liver transplant patients: a retrospective analysis. Microb Ecol. 2013;65(3):781-91. 
12. Uda A, Tanabayashi K, Fujita O, Hotta A, Yamamoto Y, et al. Comparison of whole genome amplification methods for detecting pathogenic bacteria genomic DNA using microarray. Jpn J Infect Dis. 2007;60(6):355-61.

13. Abulencia CB, Wyborski DL, Garcia JA, Podar M, Chen W, et al. Environmental whole-genome amplification to access microbial populations in contaminated sediments. Appl Environ Microbiol. 2006;72(5):3291-301.

14. Lepere C, Demura M, Kawachi M, Romac S, Probert I, et al. Whole-genome amplification (WGA) of marine photo synthetic eukaryote populations. FEMS Microbiol Ecol. 2011;76(3):513-23.

15. Oto M, Suda W, Shinoyama H. $16 \mathrm{~S}$ rRNA gene-based analysis of microbial community by whole-genome amplification and mini gel-single-strand conformation polymorphism technique. J Biosci Bioeng. 2006;102(5):482-4.

16. Wang J, Van Nostrand JD, Wu L, He Z, Li G, et al. Microarray-based evaluation of whole-community genome DNA amplification methods. Appl Environ Microbiol. 2011;77(12):4241-5

17. Wu L, Liu X, Schadt CW, Zhou J. Microarray-based analysis of subnanogram quantities of microbial community DNAs by using whole-community genome amplification. Appl Environ Microbiol. 2006;72(7):4931-41.

18. Muyzer G, de Waal EC, Uitterlinden AG. Profiling of complex microbial populations by denaturing gradient gel electrophoresis analysis of polymerase chain reaction-amplified genes coding for $16 \mathrm{~S}$ rRNA. Appl Environ Microbiol. 1993;59(3):695-700.

19. Thompson JR, Marcelino LA, Polz MF. Heteroduplexes in mixed-template amplifications: formation, consequence and elimination by 'reconditioning PCR'. Nucleic Acids Res. 2002;30(9):2083-8.

20. Ren Z, Cui G, Lu H, Chen X, Jiang J, et al. Liver Ischemic Preconditioning (IPC) improves intestinal microbiota following liver transplantation in rats through $16 \mathrm{~s}$ rDNA-based analysis of microbial structure shift. PLoS One. 2013;8(10):e75950

21. Vanhoutte T, Huys G, Brandt E, Swings J. Temporal stability analysis of the microbiota in human feces by denaturing gradient gel electrophoresis using universal and group-specific 165 rRNA gene primers. FEMS Microbiol Ecol. 2004;48(3):437-46.

22. Bartosch S, Woodmansey EJ, Paterson JC, McMurdo ME, Macfarlane GT. Microbiological effects of consuming a synbiotic containing Bifidobacterium bifidum, Bifidobacterium lactis, and oligofructose in elderly persons, determined by real-time polymerase chain reaction and counting of viable bacteria. Clin Infect Dis. 2005:40(1):28-37.

23. Hammer $\varnothing$, Harper DAT, Ryan PD. PAST: Paleontological statistics software package for education and data analysis. Palaeontologia Electronica. 2001;4(1):9. http://palaeo-electronica.org/2001_1/past/issue1_01.htm.

24. Haarman M, Knol J. Quantitative real-time PCR analysis of fecal Lactobacillus species in infants receiving a prebiotic infant formula. Appl Environ Microbiol. 2006;72(4):2359-65

25. Monstein HJ, Olsson C, Nilsson I, Grahn N, Benoni C, et al. Multiple displacement amplification of DNA from human colon and rectum biopsies: bacterial profiling and identification of Helicobacter pylori-DNA by means of 165 rDNA-based TTGE and pyrosequencing analysis. J Microbiol Methods. 2005;63(3):239-47.

26. Zaura E, Keijser BJ, Huse SM, Crielaard W. Defining the healthy "core microbiome" of oral microbial communities. BMC Microbiol. 2009;9:259.

27. Aas JA, Paster BJ, Stokes LN, Olsen I, Dewhirst FE. Defining the normal bacterial flora of the oral cavity. J Clin Microbiol. 2005:43(11):5721-32.

28. Peters BM, Jabra-Rizk MA, O'May GA, Costerton JW, Shirtliff ME. Polymicrobial interactions: impact on pathogenesis and human disease. Clin Microbiol Rev. 2012;25(1):193-213.

29. Grice EA, Segre JA. The human microbiome: our second genome. Annu Rev Genomics Hum Genet. 2012;13:151-70.

30. Zarco MF, Vess TJ, Ginsburg GS. The oral microbiome in health and disease and the potential impact on personalized dental medicine. Oral Dis. 2012;18(2):109-20.

31. Lijek RS, Weiser JN. Co-infection subverts mucosal immunity in the upper respiratory tract. Curr Opin Immunol. 2012;24(4):417-23

32. Grimaudo NJ, Nesbitt WE. Coaggregation of Candida albicans with ora Fusobacterium species. Oral Microbiol Immunol. 1997;12(3):168-73.

33. Grimaudo NJ, Nesbitt WE, Clark WB. Coaggregation of Candida albicans with oral Actinomyces species. Oral Microbiol Immunol. 1996;11(1):59-61.

34. Zhou L, Srisatjaluk R, Justus DE, Doyle RJ. On the origin of membrane vesicles in gram-negative bacteria. FEMS Microbiol Lett. 1998;163(2):223-8.
35. Corander J, Connor TR, O'Dwyer CA, Kroll JS, Hanage WP. Population structure in the Neisseria, and the biological significance of fuzzy species. J R Soc Interface. 2012;9(71):1208-15.

36. Nowotny A, Behling UH, Hammond B, Lai CH, Listgarten M, et al. Release of toxic microvesicles by Actinobacillus actinomycetemcomitans. Infect Immun. 1982:37(1):151-4.

37. Schertzer JW, Whiteley M. Bacterial outer membrane vesicles in trafficking, communication and the host-pathogen interaction. J Mol Microbiol Biotechnol. 2013;23(1-2):118-30.

38. Kuehn MJ, Kesty NC. Bacterial outer membrane vesicles and the host-pathogen interaction. Genes Dev. 2005;19(22):2645-55.

39. Lysenko ES, Ratner AJ, Nelson AL, Weiser JN. The role of innate immune responses in the outcome of interspecies competition for colonization of mucosal surfaces. PLoS Pathog. 2005;1(1):e1.

40. Sipeki N, Antal-Szalmas P, Lakatos PL, Papp M. Immune dysfunction in cirrhosis. World J Gastroenterol. 2014;20(10):2564-77.

41. Bonnel AR, Bunchorntavakul C, Reddy KR. Immune dysfunction and infections in patients with cirrhosis. Clin Gastroenterol Hepatol. 2011;9(9):727-38.

42. Chen Y, Yang F, Lu H, Wang B, Lei D, et al. Characterization of fecal microbial communities in patients with liver cirrhosis. Hepatology. 2011;54(2):562-72

43. Nasidze I, Li J, Quinque D, Tang K, Stoneking M. Global diversity in the human salivary microbiome. Genome Res. 2009;19(4):636-43.

44. Koziol-Montewka M. Microbiological causes of antibiotic therapy failure in respiratory tract infections. Pol Merkur Lekarski. 2011;30(179):373-6.

45. Harrison F. Microbial ecology of the cystic fibrosis lung. Microbiology. 2007:153(Pt 4):917-23.

\section{Submit your next manuscript to BioMed Central and take full advantage of:}

- Convenient online submission

- Thorough peer review

- No space constraints or color figure charges

- Immediate publication on acceptance

- Inclusion in PubMed, CAS, Scopus and Google Scholar

- Research which is freely available for redistribution 